\title{
Moiré-Fourier deflectometry for local heat transfer measurement over a backward-facing step
}

\author{
Gregorio L. Juste*, Efrén M. Benavides \\ Aerospace Propulsion and Fluid Mechanics, Universidad Politécnica de Madrid, Plaza Cardenal Cisneros 3, 28040 Madrid, Spain
}

\begin{abstract}
This paper explores the possibility of using the Moire-Fourier deflectometry for measuring the local heat transfer coefficient inside small confined flows (micro-channels) and their relevance for checking theoretical models. This optical technique, supplemented with a digital image processing method of fringes, is applied for studying the local heat transfer over a backward facing step. The experimental results are compared with numerical results obtained from a commercial code, which has been contrasted with relevant solutions from the literature and bulk fluid temperature measurements at the inlet and outlet sections. In order to show the possibilities of the experimental technique, the influence of assuming an adiabatic wall on the numerical heat-transfer model is examined and the degree of agreement is discussed. As a result, the paper shows that the proposed Moiré-Fourier technique is a simple experimental setup suitable for temperature measurements with an accuracy similar to the thermocouples but with a spatial resolution near $0.01 \mathrm{~mm}$.
\end{abstract}

\section{Introduction}

During the last years the compactness of the high-performance electronic systems has strongly hardened the needs associated to their cooling. As long as a high effective heat exchanger requires extracting high amounts of heat with a minimum contact surface, small channels must be used as a basic component of such systems. The downsizing of the heat exchangers is a requirement that reduces the Reynolds number, and it is well known that, when the Reynolds number is low enough (typical values for these applications are lower than 200), the flow becomes laminar. In this kind of flows, the turbulence is not present, and hence, the available heat transfer capacity is drastically reduced. It is also frequent that those channels have abrupt changes of the flow area. As a consequence of this increment of area in the flow direction, flow separation and posterior reattachment might appear. Obviously, the researcher in charge of optimizing these small channels will require an adequate experimental technique for characterizing the temperature field inside this kind of complex and small flows.

Methods based on interferometry have been broadly used to measure the temperature field in macro-scale flows, and, recently, they are being explored to investigate the heat transfer in small scale flows $[1-3]$. However, in this paper we try to study if the

\footnotetext{
* Corresponding author. Tel.: +34 913366352; fax: +34 913366375.

E-mail addresses: gregorio.lopez@upm.es, ljuste@aero.upm.es (G.L. Juste).
}

Moiré deflectometry could be a viable alternative to interferometry for this kind of flows. In this way, it has been previously shown $[4,5]$ that sufficiently accurate fringe patterns can be obtained by means of an inexpensive and simple tunable Moiré Schlieren system, and hence, the results obtained with the Moiré set-up are completely equivalent to those obtained by shearing interferometry with finite fringe alignment. In addition, if Moiré deflectometry is supplemented with a two-dimensional Fourier transform technique, the refractive index gradient map provides accurate and reliable results with high sensitivity and spatial resolution [6]. This digital image processing of Moiré fringes is especially useful in flows of very small size. Thus, it could be a good solution to the temperature measurement in small-scale flows where close-up images have to be obtained in order to increase the spatial resolution. The implementation of this experimental method to small flows has been described in Ref. [7], where the technique was applied to an external axisymmetric flow, but where a validation was not reported. Based on these ideas, the authors concluded that it could be possible to use Moiré deflectometry and Fourier mapping for temperature field measurements in $2 \mathrm{D}$ microchannels. The presented paper explains the implementation done for this purpose and the main results obtained.

The experimental technique that the authors want to investigate, combination of Moiré deflectometry with a Fourier mapping, is valid for 2D and axisymmetric flows, and hence, special care has to be taken in order to select the geometry of the channel where this experimental technique is going to be tested. 


\begin{tabular}{|llll|}
\hline \multicolumn{2}{|c|}{ Nomenclature } & $R e$ & Reynolds number \\
& & $R i$ & Richardson number \\
$D$ & distance between gratings & $R_{\mathrm{g}}$ & gas constant \\
$D_{\mathrm{h}}$ & hydraulic diameter $\left(D_{\mathrm{h}}=2 h\right)$ & $T$ & temperature \\
$G 1, G 2$ & gratings & $T_{\text {cold }}$ & cold wall temperature \\
$g$ & gravity constant & $T_{\mathrm{hot}}$ & hot wall temperature \\
$h$ & step height & $T_{0}$ & inlet fluid temperature \\
$H$ & height of the channel & $s$ & fringe shift \\
$k$ & thermal conductivity & $u$ & fluid velocity in the $x$ direction \\
$K$ & Gladstone-Dale constant & $x, y, z$ & cartesian coordinates \\
$L$ & width of the backward facing step & $X_{\mathrm{R}}$ & reattachment length \\
$L_{\mathrm{in}}$ & channel length before the step & $\beta$ & thermal expansion coefficient \\
$L_{\mathrm{d} l}$ & development length & $\delta$ & Moiré fringe deviation \\
$L_{\mathrm{out}}$ & channel length after the step & $\phi$ & deflection angle \\
$n$ & refractive index & $\varphi$ & phase change of Moiré pattern \\
$N u$ & Nusselt number & $\lambda$ & laser wave length \\
$p$ & distance between two grating lines & $v$ & kinematic viscosity \\
$P$ & pressure & $\theta$ & angle between gratings \\
$p^{\prime}$ & distance between two fringes & $\rho$ & density \\
\hline
\end{tabular}

Scientific literature has focused, both experimentally [8-10] and numerically [11,12], on the backward-facing step geometry (Fig. 1 shows a typical scheme) because it allows the researcher to study the three main effects that characterize this kind of heat exchangers at the same time: low Reynolds numbers and flow separation with very small dimensions. Published numerical studies deal with 2D simulations for steady and pulsating flows [13-15] and investigate the effects of the Reynolds number, the step height, and the frequency of the pulsating flow on the Nusselt number. Usually, the validation of the numerical simulations is performed using an experimental setup with a considerably large-aspect-ratio channel because it assures the two-dimensionality of the theoretical model. This hypothesis has been discussed by several researchers [16-20] who have investigated the effects of the duct aspect ratio by carrying out threedimensional numerical simulations for laminar and turbulent flows in rectangular ducts. These works show that, for aspect ratios larger than 16 and Reynolds numbers near 125, the 2D region becomes larger and the end-wall effects become confined in zones very adjacent to the wall. It is interesting to note that several numerical works [13-15] assume an adiabatic wall model in the region where the temperature is not controlled (i.e., the wall upstream of the step and the wall perpendicular to the flow that configures the backward-facing step), and the bottom wall downstream of the step is modeled as an uniform temperature or as an uniform heat flux. However, there are no works performed in order to investigate the influence of the adiabatic-wall assumption on the results obtained with the numerical heattransfer model. In addition, although most of the experimental

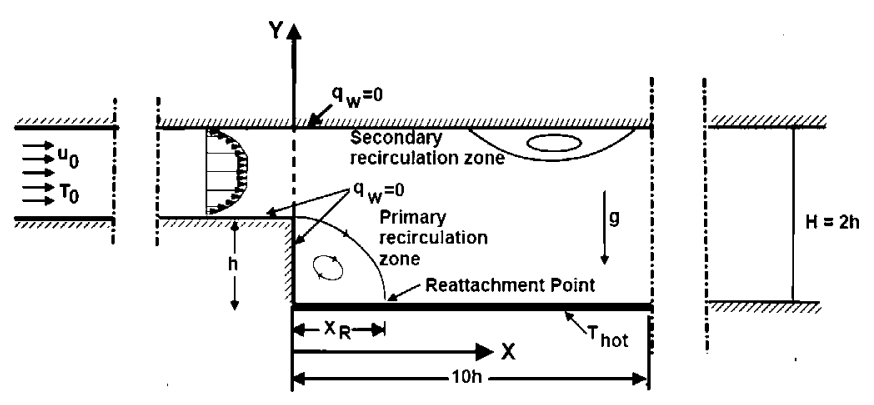

Fig. 1. Schematic diagram of a backward facing step problem. works on the backward-facing step include detailed velocity measurements $[8,10,18]$, the heat transfer is characterized only through point-wise measurements of the bulk fluid temperature with thermocouples probes at the inlet and outlet sections. From this brief revision of the available open literature, we consider that the backward-facing step is interesting for our purpose because 1) it will let show that the proposed experimental technique is able to experimentally measure the temperature field in order to derive the local heat transfer coefficient in any point of the wall, and, 2) as an immediate application, it will let experimentally validate the correctness of the adiabatic wall assumption for these flows.

Therefore, the main purpose of this paper is to prove that the Moiré-Fourier deflectometry is an experimental technique that allows measuring the temperature field downstream of a small backward facing step (characteristic length $=1 \mathrm{~mm}$ ) in the case of a two-dimensional steady and laminar flow. For this reason the paper is structured as follows. Section 2 presents the Moiré-Fourier deflectometry background. Section 3 presents the experimental setup. Section 4 introduces the main equations required for calculating the temperature field from the laser patterns in the Moire-Fourier technique. Section 5 gives the main experimental results obtained. Section 6 gives numerical calculations and uses the experimental information from the previous section to explore the validity of the adiabatic-wall assumption concluding that the better numerical solution for the proposed test bench is the one that does not assume adiabatic walls. In this section some characteristic points of the flow pattern, such as the reattachment length, are compared with previous works. Finally, main results are discussed and conclusions are presented.

\section{Moiré deflectometry background}

Moiré deflectometry is an optical method that provides quantitative data on the gradient of the density field and, in uniform pressure flows, on the temperature distribution (note that at the low Reynolds numbers explored in this paper, typically lower than 200 , the dynamic pressure is negligible when compared to the static pressure, and hence, the variations of density due to changes in the pressure are also negligible when compared to the variations of density imposed by the temperature field). In addition, this technique has shown to be less sensitive to geometrical 
misalignment and vibrations than interferometry. An excellent theoretical review of refractive index mapping by Moiré deflectometry is presented in Ref. [21].

The sensitivity of a given Moiré set-up is shown to be proportional to the ratio $D / p$, with $D$ being the inter-grating distance and $p$ the grating pitch. Thus, the sensitivity could be improved by increasing $D$ or, what is equivalent, the grating frequency. This means that, in order to obtain good spatial resolution, it is necessary to capture a Moiré pattern with several fringes, which can be accomplished by conveniently selecting the grating frequency. However, the interpretation of these fringe patterns can be difficult when the diffraction effects are present. As it is pointed out in Refs. $[22,23]$, the angular and spatial resolutions are linked to each other, so its product cannot be smaller than $\lambda / 2 \pi$. Therefore, decreasing the diffraction blur requires to choose a small value for $D$, and this increases the lower bound of the sensitivity range. In an experiment where there is a relatively large deflection angle, that is to say, a large refractive-index gradient, it is no necessary a very high sensitivity, and hence, it is possible to choose a small value of the relation $D / p$. The main advantage of this configuration is the reduction of the diffraction blur.

This technique was investigated by the authors in real 3D external small flows [24,25], where the accuracy and spatial resolution in the measurement of the flow field temperature was established as quite good: the spatial resolution was better than $0.01 \mathrm{~mm}$ with an error in the temperature (Celsius scale) lower than $5 \%$.

\section{Experimental set-up}

Fig. 2 shows the Moire deflectometry system for the temperature mapping used in the present work. The optical arrangement is quite similar to a Schlieren system, where the Schlieren head has been replaced by two equal gratings $G 1$ and $G 2$ of pitch $p$, separated by a distance $D$, and oriented with an angular difference, $\theta$, between them. The beam of a Verdi diode laser (wavelength of $532 \mathrm{~nm}$ ) was collimated and spatially filtered using a Keplerian beam expander and then directed to the test section.

After passing through the test section, the light rays reach the grating G1. The shadow of this straight line grating G1 falls onto grating G2, and the Moiré pattern is formed on a mat screen. The final pattern is collected in real time by a JVC TK-C720E camera $(752 \mathrm{H} \times 582 \mathrm{~V}$ ) with lenses for close-up magnification. The video picture was digitalized with a Pinnacle Systems frame grabber (768 $\times 576$ pixels) and stored in a personal computer for further processing. The experiments were carried out with a beam diameter in the test section of $6 \mathrm{~mm}$. With this beam size and the magnification lens, the spatial resolution was $0.01 \mathrm{~mm}$.

The gratings used for this study were Ronchi Rulings with 20 lines $/ \mathrm{mm}$. The separation between them was adjusted with a micrometer to minimize the diffraction effects $(D=23.1 \mathrm{~mm})$. The obtained images were processed, with the aid of image analysis software IJ [26], in order to enhance image quality before carrying out the data extraction from the fringe patterns.

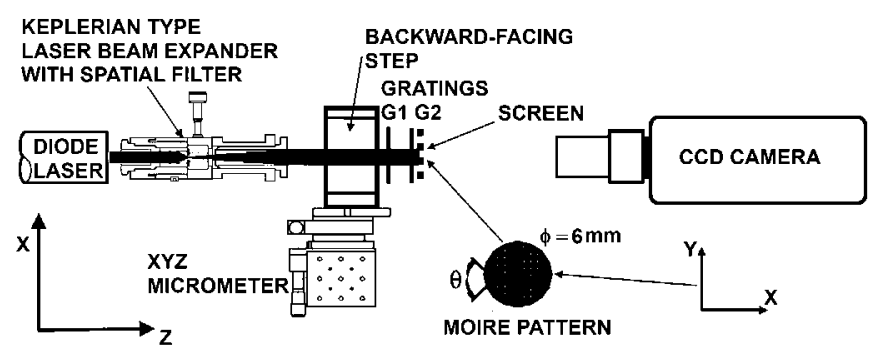

Fig. 2. Experimental set-up for Moire deflectometry.
In order to calculate the temperature corresponding to the refractive index gradient from the Moiré pattern, a phase extraction method, based on a two-dimensional fast Fourier transform (FFT), was used. The detailed mathematical model is described in Ref. [27]. In the present work, the phase analysis based on FFT was carried out with the IDEA code [28].

The geometry of the backward-facing step used in this work is similar to the one used by Terhaar et al. [29] with water. Fig. 3a and b provides a sketch of the experimental setup and the geometry of the backward facing step with the nomenclature and the main dimensions. The test facility used in this work has two glass windows, instead of PMMA windows, because the PMMA refraction index is not homogeneous and hence, a PMMA side wall deviates and changes the phase of the laser beam, which affects drastically to the accuracy of the measurements (note also that PMMA's refraction index changes with the temperature). In addition, in the test facility used in this paper, the experiments were carried out with air instead of water because the water's refraction index has a strong dependency with the temperature, which restricts the maximum temperature gradient that can be measured with Moire deflectometry. In this sense, Keren et al. [23] reported that "when considering objects with variable focal lengths one should bear in mind that Moire analysis is meaningful only when the measured quantity (refractive index in our case) does not vary too rapidly on a pitch scale. We thus treat only objects whose deviations from lens like behavior fall into one of two categories: focal length varies slowly compared with the pitch or, rapid variations of focal length are confined to regions of the order of a single period $p$, or smaller than the spatial resolution requirements".

Special care was dedicated to control the mass flowrate with the purpose of obtaining Reynolds numbers similar to the ones explored by Ref. [29], which assures a laminar flow.

The length of the inlet channel used in the presented test facility, $L_{\text {in }}$ is larger than the length obtained with the correlation provided by Durst et al. [30], who conducted a detailed numerical study and proposed the nonlinear correlation for two-dimensional channels given by $L_{\mathrm{d} 1} / h=\left[(0.619)^{1.6}+(0.0567 \mathrm{Re})^{1.6}\right]^{1 / 1.6}$. For the Reynolds number corresponding to the experimental test, the inequality $L_{\mathrm{dl}}=15.74 \mathrm{~mm}<L_{\mathrm{in}}=37 \mathrm{~mm}$ holds. This extra length ensures the fully development of the flow and hence, in addition, it assures that the flow field in the test section does not contain any additional perturbation created by the flow source.

The selected length of the channel behind the step, $L_{\text {out }}$, ensures that the outlet condition does not affect the flow near the step: Demuren et al. [31] demonstrated numerically that the flow field is not affected by increasing $L_{\text {out }} / h$ beyond a value of seven. In addition, the aspect ratio of the implemented test section is very large $(L / h=50)$, higher than the value pointed out by Iwai et al. [16] for similar Re numbers with the purpose of minimizing the three dimensional and end-wall effects. This ensures a two-dimensional flow in the step and in almost all of the channel width. The expansion ratio, defined as the channel height downstream of step divided by the step height, $H / h$, is equal to 2 .

The heated surface behind the step with a length $10 \mathrm{~h}$ is an aluminum block (thermal conductivity $=180 \mathrm{~W} \mathrm{~K}^{-1} \mathrm{~m}^{-1}$ ). The heater was a simple resistor embedded in the aluminum block, provided with an adjustable DC power supply in order to keep constant the temperature for each flow condition. The length of the resistor was approximately equal to the length of the block, so the heating power was uniformly distributed over the whole block.

The probes for discrete temperature measurements are type-K thermocouples of $0.12 \mathrm{~mm}$ diameter wire, placed in different positions of the channel as it is shown in Fig. 3a.

The air supply is obtained by means of a centrifugal blower with an adjustable rotational speed that lets set the mass airflow, which is measured by a hot-surface flow-meter. 

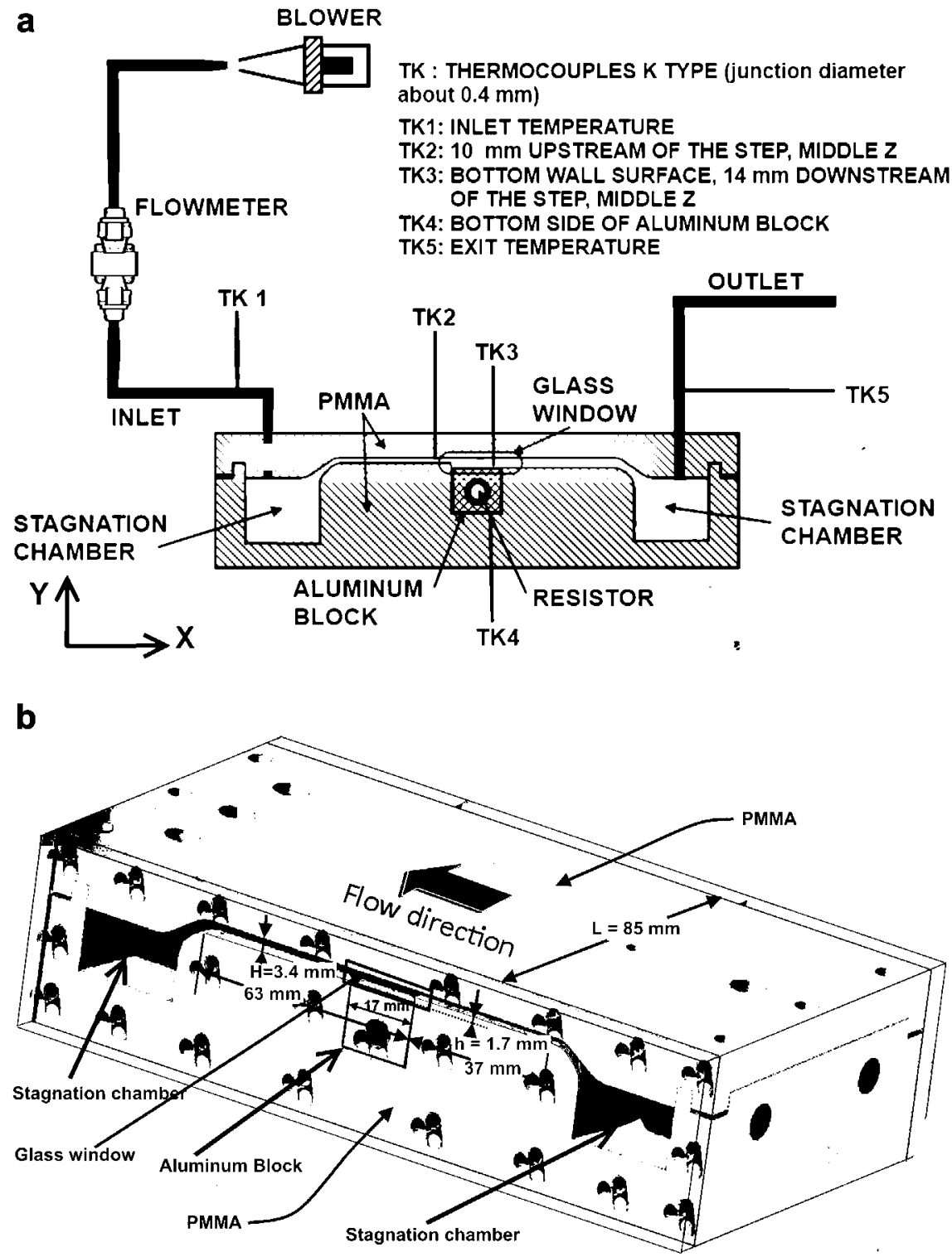

Fig. 3. Experimental facility and geometry for the backward-facing step.

The backward facing step is attached to a three-axis translation system with a micrometer with a $15 \mathrm{~mm}$ travel range, which enables accurately setting the area of the experiment and exploring the temperature field downstream of the step.

Before any measure was made, enough time was waited in order to ensure the establishment of the steady state condition (The measurements were started when no temperature variations were observed within the accuracy of the experimental setup). Later, the step was moved, using the translation system, along $x$ axis to explore $14 \mathrm{~mm}$ downstream of the step. Fig. 4 shows a typical result of the temporal evolution of the temperature measurements with the thermocouples for the experiments carried out.

\section{Evaluation of the temperature field with Moirê deflectometry}

The analysis of the temperature field in Moire deflectometry is based on the use of two images, which are obtained in the absence and presence of flow. If the fluid field is uniform, that is, without flow and with the heater off, a straight and unperturbed Moiré pattern with spatial period $p^{\prime}$ is obtained. This unperturbed solution is given by:

$$
p^{\prime}=\frac{p}{2 \sin \frac{\theta}{2}} \cong \frac{p}{\theta}
$$

Any disturbance in refractive index due to the density gradient deflects the parallel light beam and hence, the associated Moiré fringes appear distorted on the screen. The shift $s$ at each point of the fringe and the deflection angle $\phi$ are linked with the refractive index $n$ by means of the following system of equations:

$\phi=\frac{1}{n_{\infty}} \int_{0}^{L} \frac{\partial n}{\partial y} \mathrm{~d} x$

$\phi=\frac{2 s \sin (\theta / 2)}{D}$

The phase change $\varphi$ of the Moiré pattern is also related to the displacement of the fringes, and thus, to the refractive 
index gradient. These dependencies are given by the following equations:

$\varphi=\frac{2 \pi s}{p^{\prime}}$

$\varphi=\frac{2 \pi D}{p} \frac{1}{n_{\infty}} \int_{0}^{L} \frac{\partial n}{\partial y} \mathrm{~d} x$

For a two-dimensional flow field, the refractive index gradient in the fringe direction can be obtained as

$\frac{\partial n}{\partial y}=\frac{p n_{\infty}}{2 \pi D L} \varphi$

Applying the Gladstone-Dale law, the density can be obtained from the molar refractivity of the fluid in the following form:

$n-1=K \rho$

In gas flows with uniform pressure, the temperature field can be calculated through the use of the ideal gas law, if the pressure and gas composition are known $\left(R_{\mathrm{g}}\right.$ is the gas constant $)$ :

$P=\rho R_{\mathrm{g}} T$

\section{Experimental results}

The Moiré deflectometry described before, combined with a two-dimensional fast Fourier transform (FFT), was applied to the backward facing step at the following test conditions: $T_{0}=299 \mathrm{~K}$, $T_{\text {hot }}=318 \mathrm{~K}$ and flowrate $=6.5 \mathrm{~L}$ per minute. Fig. 5 shows the obtained result: Fig. $5 a$ is a drawn of the initial Moire fringe pattern of the flow next to the step, Fig. 5b shows the wrapped phase computed from the fringe pattern, and Fig. $5 \mathrm{c}$ shows the final phase map.

Fig. 6 shows the reconstruction of the temperature map downstream of the step obtained from the final phase map (fixing the reference level as the temperature measured with a thermocouple in the heated bottom wall surface). The experimental temperature field obtained with the Moiré deflectometry is compared with the numerical field (explained in the next section) in Fig. 7, where the results at several positions are plotted as a function of the distance normal to the surface of the bottom wall.

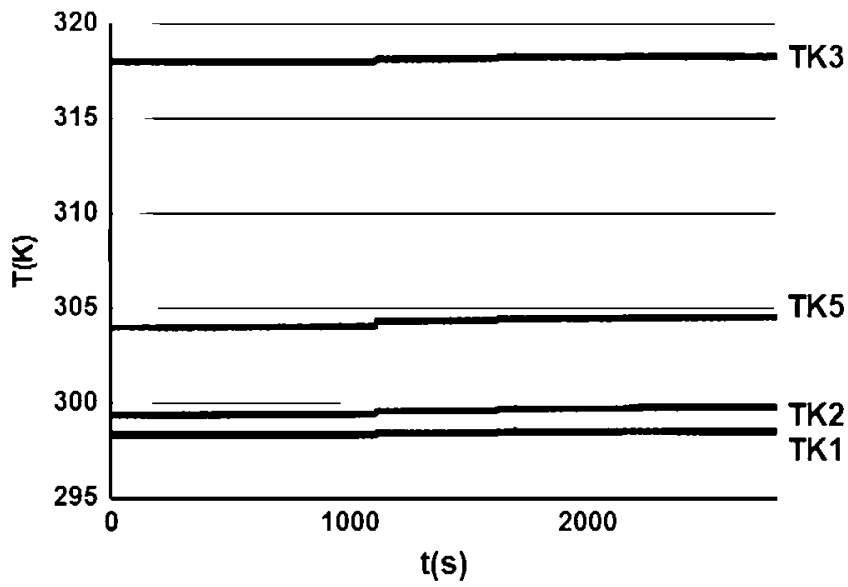

Fig. 4. Temporal evolution of the temperature signal in the middle $x y$-plane $(z=L / 2)$, previous to the stabilization of the thermocouple.

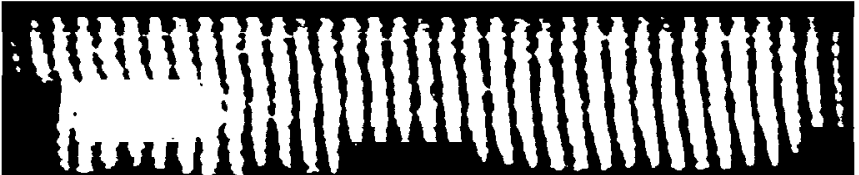

(a)

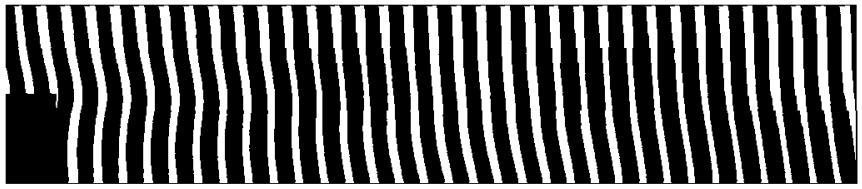

(b)

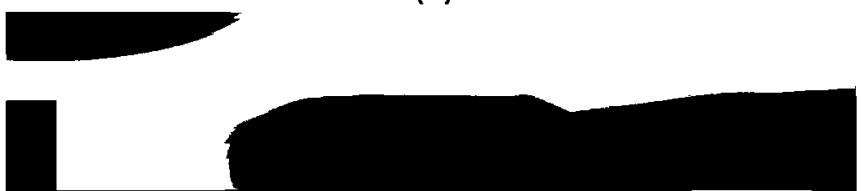

(c)

Fig. 5. Experimental result of Moiré deflectometry applied to backward-facing step $\left(R e=162\right.$ and $\left.T_{\text {hot }}=318 \mathrm{~K}\right)$. a) initial Moiré pattern; b) wrapped phase map; c) fina phase map.

\section{Numerical results}

According to the geometry of the experimental set-up and the operating test conditions, the problem studied numerically is a two-dimensional steady laminar convective flow in a duct with a backward-facing step. In order to solve the numerical problem, a commercial CFD package (ANSYS-Fluent) was used. The code is capable of solving the full Navier Stokes equations in twodimensional or three-dimensional geometries. For this purpose, the governing equations are discretized on a non-uniform Cartesian grid using a finite volume procedure [32]. A second-order upwind scheme was used for the discretization of all the equations to achieve higher order accuracy. An implicit linear equation solver was used in conjunction with an algebraic multigrid method. Velocity-pressure coupling was achieved using a SIMPLE (semiimplicit method for pressure-linked equations) algorithm and the convergence was assured by using the default under-relaxation factors. The correct prediction of the thermal field requires the inclusion of conduction effects in solids. Therefore computation of thermal conduction through solid material, coupled with heat transfer in fluid was carried out (Conjugate Heat Transfer, CHT) [32].

Fig. 8 shows the computational domain, which represents the two-dimensional section of the longitudinal straight duct of the experimental set-up. The inlet flow condition consists of an air stream with uniform profile of temperature, $T_{0}=299 \mathrm{~K}$, and velocity $u_{0}=0.75 \mathrm{~m} \mathrm{~s}^{-1}$ corresponding to a flowrate of $6.5 \mathrm{~L}$ per minute, equal to the one of the experimental test. A pressure condition was applied as the boundary condition at the exit plane (zero gauge pressure), which is far downstream of the step to reduce the influence of the outflow conditions. It was confirmed

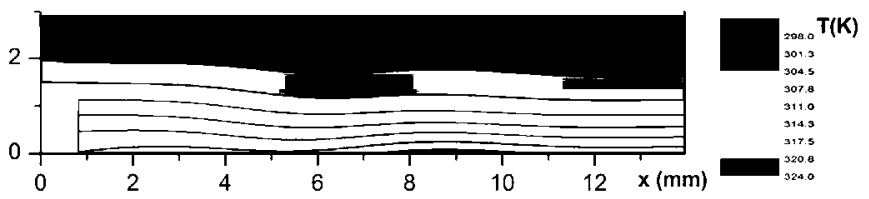

Fig. 6. Experimental isothermal lines near the step (obtained from the final phase map). 

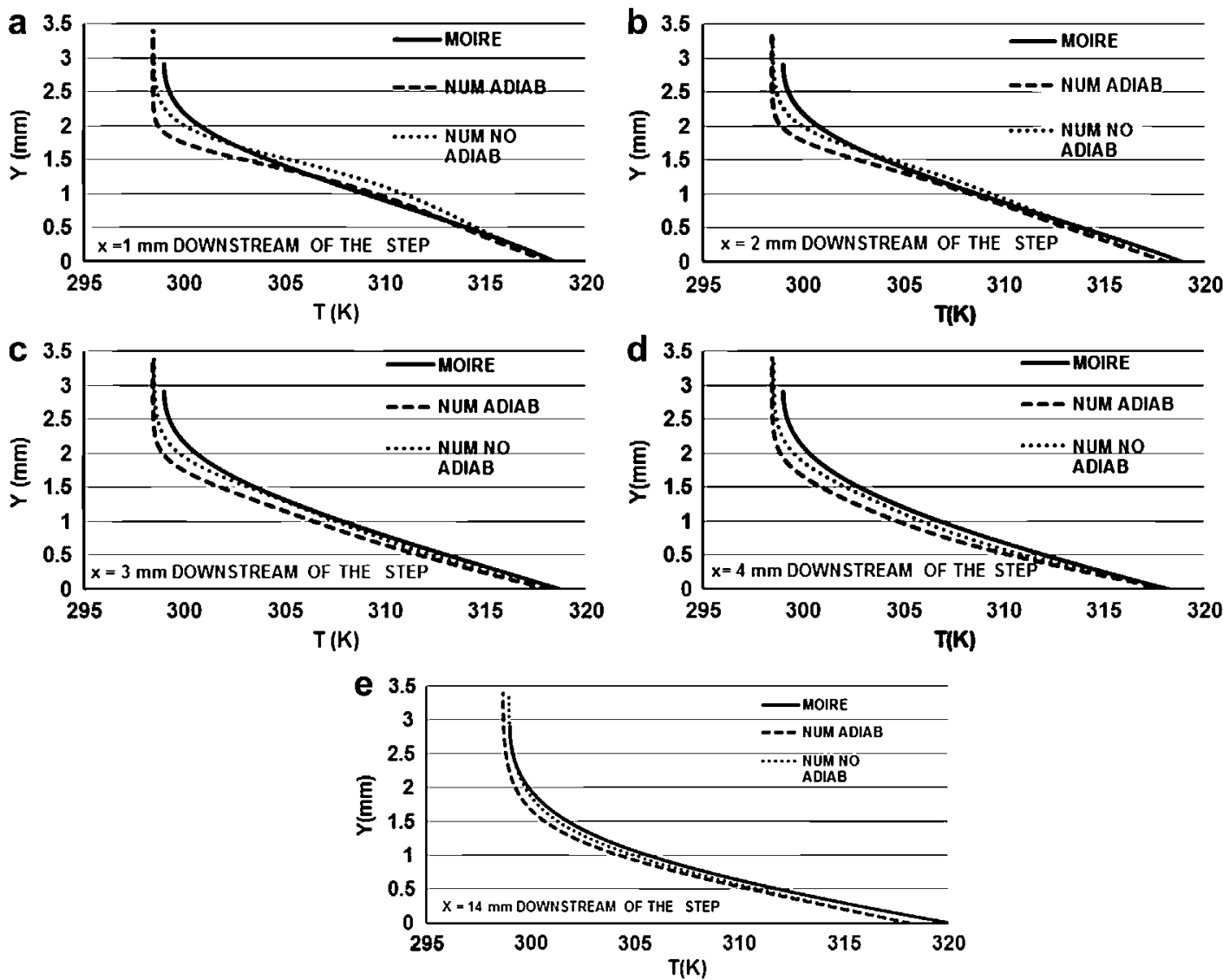

Fig. 7. Vertical temperature profiles at several positions downstream of the step. Comparison between experimental and numerical results.

that the use of a longer computational domain did not change the flow behavior in the step region. This confirms the correctness of selecting the outlet length of the channel based on the results reported by Demuren et al. [31] (see Section 4). On all solid walls noslip boundary condition was applied.

During the calculations, the temperature of the bottom wall is maintained constant (the temperature downstream of the step is fixed to $318 \mathrm{~K}$ over a length of $10 \mathrm{~h}$ ), while the facing step and the bottom wall upstream of the step are taken as adiabatic in the first simulation and non-adiabatic (with a thermal conductivity of the PMMA equal to $k=0.19 \mathrm{~W} \mathrm{~K}^{-1} \mathrm{~m}^{-1}$ ) in the second one. The top wall is assumed adiabatic in both cases. We do not consider necessary to study the change induced by the thermal conductivity of the upper wall because the top wall and the upper part of the flow have a uniform temperature field. The uniformity of the temperature field in this region was observed previously by Ref. [14], but the same result is also experimentally obtained in this paper. Fig. 6 shows that there is a fluid region near the upper wall, whose temperature is very close (less than $1{ }^{\circ} \mathrm{C}$ ) to the temperature that the wall has. This assures that the gradient of temperature in this region is negligible with independence of having a conductive material in the upper wall or not (note that the upper isothermal line in Fig. 6 is near parallel to the top wall).

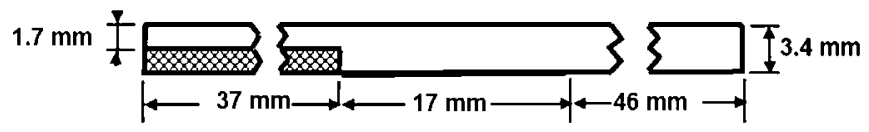

Fig. 8. Basic dimensions of the computational domain.
The Reynolds number, based on the inlet velocity and on the characteristic length $D_{\mathrm{h}}$ (hydraulic diameter $=2 h$ ) in agreement with Armally et al. [8], is:

$R e=\frac{u_{0} 2 h}{\nu}=162$

where the kinematic viscosity $\nu$ is evaluated for the air at the inlet temperature.

The Richardson number for this velocity and temperature difference between the hot and cold wall is:

$R i=\frac{g \beta\left(T_{\text {hot }}-T_{\text {cold }}\right) H}{u_{0}^{2}}=3.85 \cdot 10^{-3}$

This low number shows clearly that natural convective effects are negligible when compared to the forced convective heat transfer

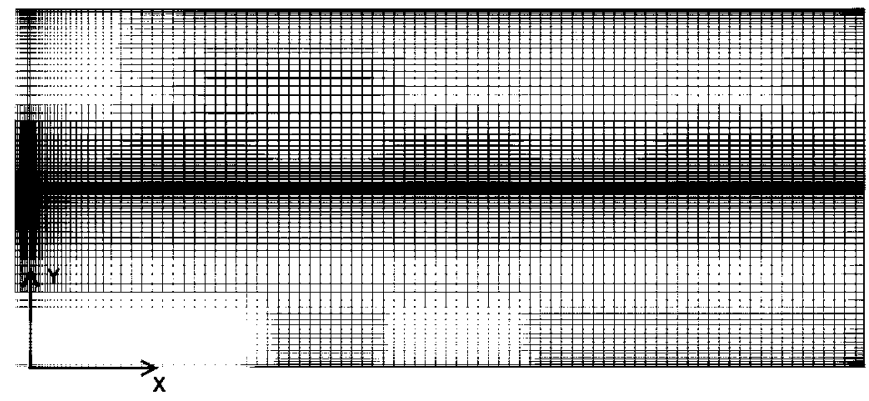

Fig. 9. Final computational mesh used, showing the refinement near the step. 
Several quadrilateral meshes were employed to achieve the mesh-independence. Finally, a mesh with 100,890 grid cells was used in the computations. The region corresponding to the step is shown in Fig. 9.

The accuracy of the numerical procedure was first validated against the benchmarked experimental results of Armaly et al. [8]. Particular attention was put in the reattachment length, which is a key measure of the computational accuracy of any numerical scheme. This parameter is the distance from the step to the position on the bottom wall at which the velocity along the channel becomes positive. Fig. 10 shows the results obtained with the code ANSYS-Fluent for the experimental test conditions $(R e=162)$ as well as for another randomly selected condition $(R e=100)$ and for the case presented by Armaly et al. [8]. As it can be seen in that figure, the agreement is good.

Samples of the results obtained with the numerical simulation at the experimental test conditions are shown in Figs. 11 and 12. Fig. 11 compares the isotherms for the two hypotheses, adiabatic and non-adiabatic. Fig. 12 presents the streamlines and shows the general steady flow features for the backward facing step. Both results evidence also good agreement with those shown by Khanafer et al. [14] for steady state.

\section{Discussion}

The agreement between the results obtained with Moiré-Fourier deflectometry and the numerical results is quite satisfactory near the wall but discrepancies appear far from the bottom wall. There are two plausible reasons for this: 1 ) the sensitivity of the Moiré set-up was adjusted to be able to capture the temperature gradient near the wall, and therefore, the sensitivity is lost in other parts of the flow field where the temperature is slightly perturbed [24], and 2) the numerical integration of the gradient of refraction along the $y$ axis has a numerical error that increases far from the bottom wall. The experimental temperature profile agrees better with the numerical results that come from the computations carried out with the hypothesis of non-adiabatic step walls than with the ones obtained from using the typical hypothesis of adiabatic walls, except, in the very close region to the facing step $(x=1 \mathrm{~mm})$. (Note that this uncertainty in a very close region to the step results from the diffraction effects introduced by the side wall of the step.)

The results obtained show that it can be convenient to consider the conductivity in the numerical models, and this with

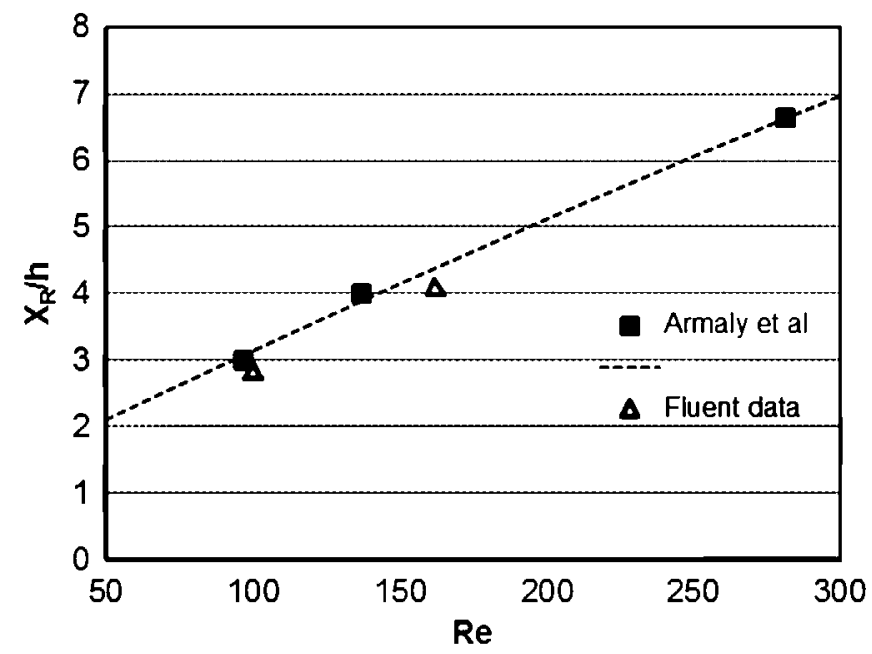

Fig. 10. Reattachment length. Comparison between the present numerical study and that of Armaly et al. [8].

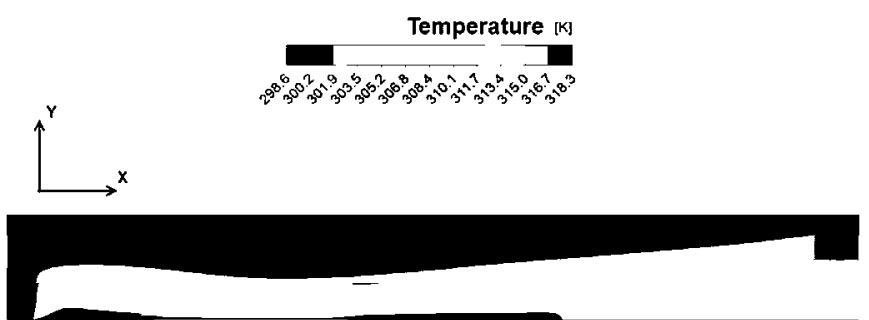

(a)

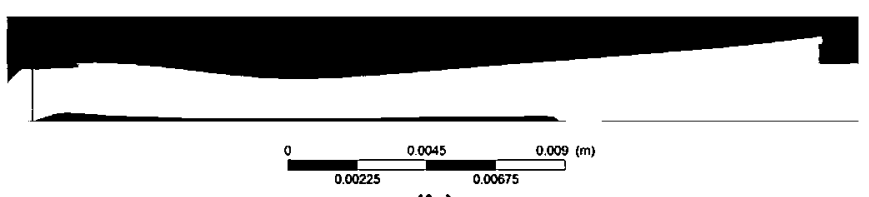

(b)

Fig. 11. Numerical isothermal lines ( $R e=162$ and $T_{\text {hot }}=318 \mathrm{~K}$ ). a) Adiabatic assumption, and b) non-adiabatic assumption.

independency of using a low thermal conductivity in the material employed for manufacturing the experimental setup. The discrepancy between the two models, adiabatic and non-adiabatic, can be corroborated from the curves shown in Fig. 13. This figure shows the local Nusselt number in the bottom wall, which is a dimensionless measure of the local heat transfer, $k \partial T / \partial y$, where the variation of the transversal coordinate is made dimensionless with the hydraulic diameter $D_{\mathrm{h}}=2 h$, and the variation of the temperature is made dimensionless with the difference between the bulk temperature and the wall temperature:

$$
\begin{aligned}
& N u=\left.\frac{2 h}{T_{\mathrm{b}}(x)-T_{\text {wall }}} \frac{\partial T}{\partial y}\right|_{\text {wall }} \\
& T_{b}(x)=\frac{\int_{0}^{H} \rho(x, y) u(x, y) T(x, y) d y}{\int_{0}^{H} \rho(x, y) u(x, y) d y}
\end{aligned}
$$

Moreover, the comparison of the mass-weighted average temperature at the channel exit (note that exit plane is located at $x=100 \mathrm{~mm}$ ), calculated as $\bar{T}_{\text {numerical }}(x)=$ $\int_{0}^{H} \rho(x, y) u(x, y) T(x, y) \mathrm{d} y / \int_{0}^{H} \rho(x, y) u(x, y) \mathrm{d} y$, confirms that the numerical model with non-adiabatic step walls agrees better with the experimental measurements than the adiabatic one: $T_{\text {numerical_adiabatic }}=302.9 \mathrm{~K}, T_{\text {numerical_non-adiabatic }}=303.4 \mathrm{~K}$, and $T_{\text {thermocouple }}=304.3 \mathrm{~K}$. Therefore, these effects should be taken into

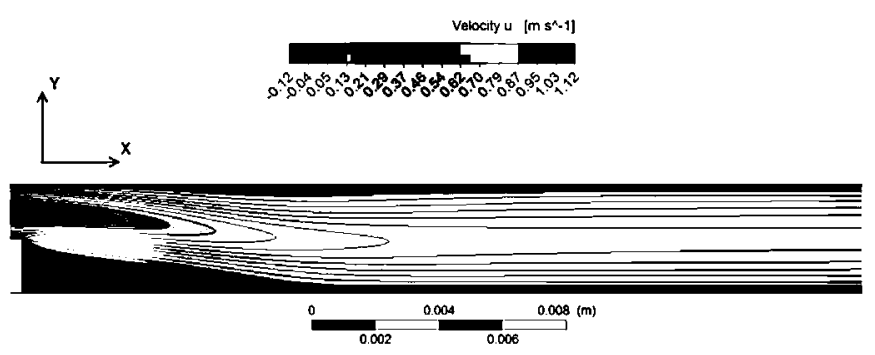

Fig. 12. Lines of constant horizontal velocity $\left(R e=162\right.$ and $\left.T_{\text {hot }}=318 \mathrm{~K}\right)$, calculated assuming a non-adiabatic wall. 


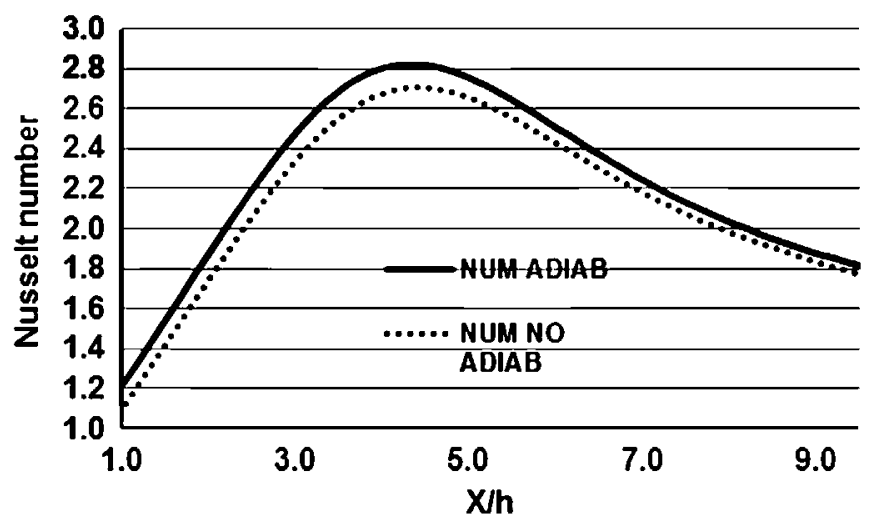

Fig. 13. Effect of adiabatic and non-adiabatic assumptions on the local Nusselt number downstream of the step $\left(R e=162\right.$ and $\left.T_{\text {hot }}=318 \mathrm{~K}\right)$. Coordinates correspond to Fig. 1 .

account in the numerical models, mainly in the studies where the effect of the investigated parameters, based in global measurements (inlet and outlet temperatures) are of the same order that the influence of the adiabatic hypothesis.

From the results, we can assure that the proposed MoiréFourier technique is suitable for attaining a measurement accuracy similar to the thermocouples but with a spatial resolution near $0.01 \mathrm{~mm}$. This level of spatial resolution is difficult for a classical technique based on thermocouples. Therefore, the experimental results show the potential of Moire deflectometry for heat transfer measurements in flows confined in small geometries, albeit of the possible error sources in the Moiré technique previously studied by the authors [24,25], such as, 1 ) end walls effects, 2) phase map reconstruction process, and phase errors introduced by non-linear response of CCD detector. Typically the most important source of error is the first one because the temperature error could be as high as $1 \%$ for a case where the difference of temperature is $60 \mathrm{~K}$ and where the ratio between the thermal boundary layer thickness and the backward facing step width, $L$, is 0.1 . The second source of error is near $0.4 \%$ and independent of the temperatures studied.

\section{Conclusions}

The temperature field for a two-dimensional backward facing step at laminar steady flow regime has been experimentally determined by means of the combination of Moire deflectometry and a two-dimensional Fourier transform. The results have been obtained with a conventional setup and show the ability of this experimental technique for measuring the temperature field in small confined flows with a spatial resolution about $0.01 \mathrm{~mm}$, and hence, it has potential for determining the local heat transfer in the regions of concern inside the flow.

Additionally, the comparison of the experimental result with several numerical solutions shows up the convenience of considering the actual conductivity of the walls. In this work, a comparison between the temperature distribution considering an adiabatic wall, which is the usual approach, and the temperature distribution coming from assuming a non-adiabatic wall (using a conjugate heat transfer model for specifying the boundary conditions of the numerical model) was done. Without this correction, the numerical results and the experimental ones have discrepancies of the same order that the effect of the backward-facing step itself has.

\section{References}

[1] V. Sajith, C.B. Sobhan, Digital interferometric measurement of forced convection heat transfer in a miniature rectangular channel, Exp. Heat Transfer 21 (2008) 314-333.

[2] D. Newport, C.B. Sobhan, J. Garvey, Digital interferometry: techniques and trends for fluid measurement, Heat Mass Transfer 44 (2008) 535-546.

[3] V. Sajith, Divya Haridas, C.B. Sobhan, G.R.C. Reddy, Convective heat transfer studies in macro and mini channels using digital interferometry, Int. J. Therm. Sci. 50 (2011) 239-249.

[4] O. Kafri, Non-coherent method for mapping phase objects, Opt. Lett. 5 (1980) $555-557$.

[5] J. Stricker, O. Kafri, A new method for density gradient measurements in compressible flows, AIAA J. 20 (1981) 820-823.

[6] J. Stricker, E. Keren, O. Kafri, Axisymmetric density field measurements by Moiré deflectometry, AIAA J. 21 (1983) 1767-1769.

[7] S. Yaozu, Z. Xiangchun, Z. Honglin, Laser Moiré deflectometry applicable for mini-micro-scale flow visualization, Proc. SPIE 5058(2003). http://dx.doi.org/ $10.1117 / 12.509775$.

[8] B.F. Armaly, F. Dursts, J.C.F. Pereira, Experimental and theoretical investigation of backward-facing step flow, J. Fluid Mech. 127 (1983) 473-496.

[9] T. Lee, D. Mateescu, Experimental and numerical investigation of 2-D backward-facing step flow, J. Fluid Struct. 12 (1998) 703-716.

[10] N. Furuichi, T. Hachiha, M. Kumada, An experimental investigation of a largescale structure of a two-dimensional backward-facing step by using advanced multi-point LDV, Exp. Fluids 36 (2004) 274-281.

[11] T.P. Chiang, T.H. Sheu, A numerical revisit of backward-facing step flow problem, Phys. Fluids 11 (1999) 862-874.

[12] G. Biswas, M. Breuer, F. Durst, Backward-facing step flows for various expansion ratios at low and moderate Reynolds numbers, J. Fluids Eng. 126 (2004) 363-374.

[13] A. Valencia, L. Hinojosa, Numerical solutions of pulsating flow and heat transfer characteristics in a channel with a backward-facing step, Heat Mass Transfer 32 (1997) 143-148.

[14] K. Khanafer, B. Al-Azmi, A. Al-Shammari, I. Pop, Mixed convection analysis of laminar pulsating flow and heat transfer over a backward-facing step, Int. J. Heat Mass Transfer 51 (2008) 5785-5793.

[15] E.M. Benavides, Heat transfer enhancement by using pulsating flows, J. Appl. Phys. 105 (2009).

[16] H. Iwai, K. Nakabe, K. Suzuki, Flow and heat transfer characteristics of backward-facing step laminar flow in a rectangular duct, Int. J. Heat Mass Transfer 43 (2000) 457-471.

[17] J.H. Nie, B.F. Armaly, Reattachment of three-dimensional flow adjacent to backward-facing step, J. Heat Transfer 125 (2003) 422-428.

[18] J.H. Nie, B.F. Armaly, Reverse flow regions in three-dimensional backwardfacing step flow, Int. J. Heat Mass Transfer 47 (2004) 4713-4720.

[19] J.G. Barbosa, N.K. Anand, V. Sarin, Numerical simulation of mixed convective flow over a three-dimensional horizontal backward facing step, J. Heat Transfer 127 (2005) 1027-1036.

[20] H. Lan, B.F. Armaly, J.A. Drallmeier, Three-dimensional simulation of turbulent forced convection in a duct with backward-facing step, Int. J. Heat Mass Transfer 52 (2009) 1690-1700.

[21] O. Kafri, I. Glatt, The Physics of Moiré Metrology, John Wiley and Sons, New York, 1990, pp. 89-109, 136-149.

[22] E. Bar-Ziv, Effect of diffraction on the Moire image, J. Opt. Soc. Am. A 2 (1985) $371-379$.

[23] E. Keren, O. Kafri, Diffraction effects in Moiré deflectometry, J. Opt. Soc. Am. A 2 (1985) 111-120.

[24] G.L. Juste, E.M. Benavides, Temperature measurement in small-scale flows with digital Moiré deflectometry, Exp. Heat Transfer 24 (2011) 201-214.

[25] G.L. Juste, E.M. Benavides, End-wall errors in temperature measurement of external convective heat transfer with Moiré deflectometry, Opt. Lasers Eng. 49 (2011) 8-12.

[26] Image Processing and Analysis in Java. Version 1.42, 2009. http://rsb.info.nih. gov $/ \mathrm{ij} /$.

[27] S. Nicola, P. Ferraro, I. Gurov, R. Koviazin, M. Volkov, Fringe analysis for Moiré interferometry by modification of the local intensity histogram and use of a two-dimensional Fourier transform method, Meas. Sci. Technol. 11 (2000) 1328-1334.

[28] M. Hirp, P. Reitere, IDEA: Software for Interferometrical Data Evaluation, Institut für Experimental Physik Technische, Universität Graz, Graz, Austria, 1999.

[29] S. Terhaar, A. Velázquez, J. Arias, M. Sánchez-Sanz, Experimental study on the unsteady laminar heat transfer downstream of a backward facing step, Int. Commun. Heat Mass Transfer 37 (2010) 457-462.

[30] F. Durst, S. Ray, B. Unsal, O.A. Bayoumi, Development lengths of laminar pipes and channel flows, ASME J. Fluids Eng. 127 (2005) 1154-1160.

[31] A. Demuren, R. Wilson, Estimating uncertainty in computations of twodimensional separated flows, J. Fluids Eng. 116 (1994) 216-220.

[32] Fluent Inc., Fluent 6.3 User's Guide, Ansys, Inc., Southpointe, Canonsburg, PA. 